\title{
Quantum-like modeling: cognition, decision making, and rationality
}

\section{Andrei Khrennikov ${ }^{1}$}

Received: 16 December 2019 / Accepted: 11 February 2020 / Published online: 9 November 2020 (c) The Author(s) 2020

Recent years were characterized by the real explosion of interest to applications of quantum measurement theory and other parts of quantum formalism outside of physics, especially in psychology, decision making, economics, finances, and social science (as well as in genetics and molecular biology), see, e.g., monographs (Khrennikov 2004, 2010; Busemeyer and Bruza 2012; Haven 2013; Asano et al. 2015; Bagarello 2019; Schade 2019). Quantum-like models reflect those specialties of cognitive information processing which match well with the quantum formalism. We emphasize that the strategy of quantum-like modeling does not assume that the whole body of quantum theory should be explored. The quantum-like representation of mental states and (self-)observations is operational. It does not provide the complete picture of cognitive processes including functioning of neuronal networks in the brain. In principle, one can search for finer models, so to say prequantum mod$e l s$, and couple such models with the quantum operational description.

The main distinguishing feature of quantum-like modeling is exploring quantum probability theory, the calculus of complex probability amplitudes. As was shown by the author (Khrennikov 2004, 2010), one of the consequences of this calculus is modification of the formula of total probability. The classical formula, for two observables A and B (represented by random variables on the Kolmogorov probability) space has the form:

$$
\mathrm{P}(\mathrm{B}=\mathrm{y})=\sum \mathrm{P}(\mathrm{A}=\mathrm{x}) \mathrm{P}(\mathrm{B}=\mathrm{y} \mid \mathrm{A}=\mathrm{x}) .
$$

Its quantum analog is characterized by the appearance of the additional term, a kind of the interference term. In the case of dichotomous observables, this formula has the form:

Andrei Khrennikov

andrei.khrennikov@Inu.se

1 International Center for Mathematical Modeling in Physics and Cognitive Sciences, Linnaeus

University, 35195 Växjö, Sweden 


$$
\begin{aligned}
\mathrm{P}(\mathrm{B}=\mathrm{y}) & =\mathrm{P}(\mathrm{A}=-1) \mathrm{P}(\mathrm{B}=\mathrm{y} \mid \mathrm{A}=-1)+\mathrm{P}(\mathrm{A}=+1) \mathrm{P}(\mathrm{B}=\mathrm{y} \mid \mathrm{A}=+1) \\
& +2 \cos \theta \sqrt{ }[\mathrm{P}(\mathrm{A}=-1) \mathrm{P}(\mathrm{B}=\mathrm{y} \mid \mathrm{A}=-1) \mathrm{P}(\mathrm{A}=+1) \mathrm{P}(\mathrm{B}=\mathrm{y} \mid \mathrm{A}=+1)]
\end{aligned}
$$

If $\cos \theta>0$, we get constructive interference of events, if $\cos \theta<0$, we get destructive interference of events, if $\cos \theta=0$, then the classical formula is applicable. From this viewpoint, the use of the quantum probability calculus is just accounting of deviations from classical probability theory. (In some sense, there is nothing mystical in the possibility of such perturbations). Since the "angle parameter" varies continuously, quantum probability is smoothly connected with Kolmogorov probability. We remark that generally this angle parameter has the purely probabilistic meaning and it has no relation to space geometry (although in physics, such relation can be established).

The classical probabilistic derivation of the formula of total probability is based on the existence of the probability measure that is additive and the use of the Bayes formula for probability update. In quantum theory, both assumptions are violated. There exist incompatible observables, they cannot be jointly determined and their joint probability distribution (JPD) does not exist. The textbook examples of such observables are position and momentum observables. The existence of incompatible observables is connected with the Heisenberg uncertainty principle and it is formalized in the form of the Bohr complementarity principle.

In particular, the Bayes formula is based on the operation of conjunction of events, so it preassumes the existence of JPD. To accommodate the update of probability and probability inference to observables without JPD, quantum formalism modifies the definition of conditional probability. It is based on quantum state transformation as back-action of measurement. One can consider the quantum probability calculus as non-Bayesian inference theory. For compatible observables (given by commuting Hermitian operators), quantum and classical probability inferences coincide. Thus by applying the quantum calculus outside of physics, one has to search for incompatible observables and then to represent these observables by non-commuting operators. The latter (construction of operator representation) is the separate (and complex) problem. Classically events are represented by Boolean algebra; in the quantum framework, events are represented by partial Boolean algebra, a collection of consistently coupled Boolean algebras.

From the logic viewpoint, quantum-like decision-making is based on nonclassical (non-Boolean) logic. Therefore, it is not surprising that such decision making can lead to violation of the basic postulates of normative decision theory, e.g., the Savage Sure Thing Principle. The latter is typically used as the axiom of rationality of agents. Another striking deviation is violation of Aumann's theorem. We recall that Aumann demonstrated that rational agents with common knowledge of each other's beliefs cannot agree to disagree [see (Khrennikov and Basieva 2014)]. Quantum agents, i.e., people using quantum logic (instead of Boolean logic) and, hence, quantum update of probability (instead of Bayesian update) can agree to disagree, even with common knowledge of each other's beliefs.

Quantum agents are irrational (from the viewpoint of classical rationality). However, their behavior is adapted to the surrounding environment (both physical and 
mental). Therefore, may be human's rationality has to be identified with quantum (non-Boolean) rationality.

In quantum-like modeling, a decision maker (an individual or a social system) can be considered as an open quantum information system; decisions are modelled as stationary states of the master equation describing interactions (including purely informational) between a system and its environment-decision making as decoherence. The decision theory based on open quantum systems belongs to the class of nonrational decision theories. We can treat quantum rationality as a sort of ecological rationality. The environment that is mathematically described in terms of quantum information theory does part of the work for the heuristic. Heuristics is adapted to the environment and the processes of adaptation is modelled as quantum differentiation (Asano et al. 2017).

Finally, we make a few remarks about interpretation of the quantum formalism, in physics and outside, e.g., in cognitive science, decision making, psychology. The present situation in quantum physics is characterized by huge diversity of interpretations. The recent quantum information revolution generated a bunch of new interpretations, so called information interpretations: the Zeiliner-Brukner interpretation, QBism (Fuchs and Schack), the Växjö interpretation (Khrennikov), reality without realism (Plotnitsky). By the Växjö interpretation, the quantum theory is one special version of the contextual probabilistic theory for measurements. It is close to the Bohr's interpretation (instead the notion "context", Bohr used the notion "experimental conditions'). In contrast to the Bohr interpretation, by the Växjö interpretation one can in principle go beyond the complementarity principle and construct subquantum models providing the causal presentation of natural phenomena. This interpretation is very convenient for applications outside of physics. It leads to the following.

\section{Quantum-like paradigm (Khrennikov 1999):}

The mathematical formalism of quantum information and probability theories can be used to model behavior not only of genuine quantum physical systems, but all context-sensitive systems, e.g., humans. Contextual information processing cannot be based on complete resolution of ambiguity. Therefore such systems process ambiguities, process superpositions of alternatives.

Funding Open access funding provided by Linnaeus University.

Open Access This article is licensed under a Creative Commons Attribution 4.0 International License, which permits use, sharing, adaptation, distribution and reproduction in any medium or format, as long as you give appropriate credit to the original author(s) and the source, provide a link to the Creative Commons licence, and indicate if changes were made. The images or other third party material in this article are included in the article's Creative Commons licence, unless indicated otherwise in a credit line to the material. If material is not included in the article's Creative Commons licence and your intended use is not permitted by statutory regulation or exceeds the permitted use, you will need to obtain permission directly from the copyright holder. To view a copy of this licence, visit http://creativecommons.org/licen ses/by/4.0/. 


\section{References}

Asano M, Basieva I, Khrennikov A, Ohya M, Tanaka Y (2017) A quantum-like model of selection behavior. J. Math. Psychol. 78:2-12

Asano M, Khrennikov A, Ohya M, Tanaka Y, Yamato I (2015) Quantum adaptivity in biology: from genetics to cognition. Springer, Berlin

Bagarello F (2019) Quantum concepts in the social ecological and biological sciences. Cambridge University Press, Cambridge

Busemeyer JR, Bruza PD (2012) Quantum models of cognition and decision. Cambridge University Press, Cambridge

Haven E, Khrennikov A (2013) Quantum social science. Cambridge University Press, Cambridge

Khrennikov A (1999) Classical and quantum mechanics on information spaces with applications to cognitive, psychological, social and anomalous phenomena. Found Phys 29:1065-1098

Khrennikov A (2004) Information dynamics in cognitive, psychological, social, and anomalous phenomena. Series: fundamental theories of physics. Kluwer, Dordreht

Khrennikov A (2010) Ubiquitous quantum structure: from psychology to finances. Springer, Berlin

Khrennikov A, Basieva I (2014) Possibility to agree on disagree from quantum information and decision making. J. Math. Psychol. 62(3):1-5

Schade CD (2019) Free will and consciousness in the multiverse: physics, philosophy, and quantum decision making. Springer, Berlin

Publisher's Note Springer Nature remains neutral with regard to jurisdictional claims in published maps and institutional affiliations. 